\title{
SHOULD WE FEAR QUANTUM TORMENT?
}

\author{
István Aranyosi
}

\begin{abstract}
The prospect, in terms of subjective expectations, of immortality under the no-collapse interpretation of quantum mechanics is certain, as pointed out by several authors, both physicists and, more recently, philosophers. The argument, known as quantum suicide, or quantum immortality, has received some critical discussion, but there hasn't been any questioning of David Lewis's point that there is a terrifying corollary to the argument, namely, that we should expect to live forever in a crippled, more and more damaged state, that barely sustains life. This is the prospect of eternal quantum torment. Based on some empirical facts, I argue for a conclusion that is much more reassuring than Lewis's terrible scenario. ${ }^{1}$
\end{abstract}

\section{From Quantum Immortality to Quantum Torment}

The prospect, in terms of subjective expectations, of immortality under the many-worlds interpretation of quantum mechanics is certain, as pointed out by several authors ${ }^{2}$. The argument goes as follows. If there are no collapses of the wavefunction, then the actual world is branching, as time passes, into several parallel

1 I would like to thank audiences at Boğazici University (Istanbul) and Central European University (Budapest) for valuable feedback on the main ideas of this paper. I would also like to acknolwedge the continued financial support for my research by TÜBITAK (The Scientific and Technological Research Council of Turkey) over the last 2 years.

2 For example: Euan J. Squires, The Mystery of the Quantum World (Bristol Institute of Physics Publishing, 1986), Hans P. Moravec, Mind Children: The Future of Robot and Human Intelligence (Cambridge Harvard University Press, 1988), pp. 188-190, H. Dieter Zeh, The Physical Basis of the Direction of Time (Berlin: Springer Verlag 1992), Huw Price, Time's Arrow and Archimedes' Point (Oxford: Oxford University Press 1996), pp. 221-222, Max Tegmark, 'The Interpretation of Quantum Mechanics: Many Worlds or Many Words?', Fortschritte der Physik 46 (1998), pp. 855-862, Peter Lewis, 'What is it Like to Be Schrödinger's Cat?', Analysis 60 (2000), pp. 22-29, and David K. Lewis, 'How Many Lives has Schrödinger's Cat?', The Jack Smart Lecture, Canberra, 27 June 2001, Australasian Journal of Philosophy 82 (2004), pp. 3-22. The thought experiment is known as 'quantum suicide', first proposed by physicists in 1980s, then widely discussed by several philosophers starting with Peter Lewis's 'What it is like...'. 
worlds according to the space of possible quantum states of the world that are in superposition as reflected by the time-dependent Schrödinger equation, each of these branches being equally real and actual ${ }^{3}$. The outcome of life-threatening situations are no less governed by quantum mechanical processes as everything else in our universe; 'we are all Schrödinger's cats', to use David Lewis's ('How Many Lives', pp. 18-19) formula. Such situations correspond to branchings of the actual world into a branch on which the subject of the situation dies and at least one other branch on which the subject is alive. If we are interested in what our subjective expectation about survival should be in such life-and-death branchings, then the set of branches that are relevant to the computation does not include those in which we don't survive. Since being dead is not compatible with having any experience at all, the space of branches of actuality that we have to distribute probabilities over when inquiring into what experience to expect in the future is the space of those on which we are alive. So, however small the probability of surviving a life-and-death quantum branching, we will always find ourselves on the side of life and never on the side of death. Hence, since the number of such increasingly improbable survival branches is infinite, given that the probability of surviving never drops to zero, we should expect to be alive forever. This is what we call 'quantum immortality'.

This is good news for those who value longevity, but David Lewis ('How Many Lives', p. 20) argues that:

A terrifying corollary has gone unmentioned. As well as lifeand-death branchings, there may be life-and-life branchings such that you suffer harm on some branches and not on others. In some of these branchings, the harm branches get the lion's share of the total intensity. The intensity rule applies, so you should predominantly expect to find yourself harmed. As you survive deadly danger over and over again, you should also expect to suffer repeated harms. You should expect to lose your loved ones, your eyes and limbs, your mental powers, and your health.

Born's Rule assigns probabilities, whose numerical values are given by the squared moduli of the probability amplitudes in Scrödinger's equation, to these possible states, but that does not make any of them less actual. This tension between the idea of states being both actual and probable is taken as the chief weakness of the many-worlds interpretation of quantum mechanics. 
What Lewis refers to as the intensity rule is that one should distribute expectations over branches according to their intensities in a way that would match the predictions of quantum mechanics regarding the observational outcomes of collapses, i.e. in a way to match Born's Rule (see my footnote 2). Once the death branches have been eliminated from the space of possible outcomes, we should subjectively expect to live forever, given that all branchings are life-and-life branchings, but given that all these branchings happen in the vicinity of death, we should expect to be predominantly harmed, since being harmed in life-threatening situations is very probable, hence it gets the lion's share of the total intensity of possible survival scenarios. We should, therefore, expect something like eternal torment. This is Lewis's terrifying corollary, and he expresses a genuine personal concern for his own future at the end of his article.

\section{From the Terrifying Corollary to the Comforting Corollary}

There have been a number of critical discussions dedicated to various aspects of the argument for quantum immortality and its basis, the many-worlds interpretation ${ }^{4}$, but, to my knowledge, no one has so far questioned David Lewis's terrifying corollary, or, more exactly, that that is the right implication of quantum immortality. So it looks as though there is agreement that if the argument for immortality is sound, then, by that argument's assumptions, the terrifying corollary of quantum torment follows, so we should be very concerned about our future. It is this move that I would like to question in this paper, and argue that, in fact, something almost opposite to torment is to be expected, given Lewis's assumptions.

I start by noting that the reason death branches are to be discarded when evaluating the intensities of various branches of

4 For instance, David Papineau, 'Why You don't Want to Get in the Box with Schrödinger's Cat', Analysis 63 (2003), pp. 51-58, David Papineau, 'David Lewis and Schrödinger's Cat', Australasian Journal of Philosophy 82 (2004), pp. 153-169, Paul Tappenden, 'The Ins and Outs of Schrödinger's Cat Box: A Response to Papineau', Analysis 64 (2004), pp. 157-164, Hilary Greaves, 'Understanding Deutsch's Probability in a Deterministic Multiverse', Studies in History and Philosophy of Modern Physics 35 (2004), pp. 423-456, Milan Ćirković, 'Is Quantum Suicide Painless? On an Apparent Violation of the Principal Principle', Foundations of Science 11 (2006), pp. 287-296. 
my future is that being dead is not an experiential state at all. 'Death is oblivion', as Lewis ('How Many Lives', p. 17) puts it. So the variable that is responsible for both the new event space (lacking death branches) and for the high likelihood of future suffering within that space is consciousness, not life as such. The experience of suffering requires a minimal level of selfawareness and various cognitive functions. Life, on the other hand, can also be lived in coma or in a persistent vegetative state. Coma is understood in medical science as a state with no consciousness whatsoever and from which the patient cannot be aroused, whereas the vegetative state is 'absence of responsiveness and awareness due to overwhelming dysfunction of the cerebral hemispheres, with sufficient sparing of the diencephalon and brain stem to preserve autonomic and motor reflexes and sleep-wake cycles. Patients may have complex reflexes, including eye movements, yawning, and involuntary movements to noxious stimuli but show no awareness of self or environment'. (Merck Manual Online Edition 2008)

Lewis's description of the torment you should expect refers to surviving with enough of you to sustain life, not to surviving with enough of you to sustain self-awareness; here is a quote ('How Many Lives', p. 20):

'What does matter is that the overwhelming share of the total intensity goes to branches on which less than all of you, in fact a lot less than all of you, in fact only just barely enough of you to sustain life, reappears. Much the same goes for all the other deadly dangers that we face.

What you should predominantly expect, if the no-collapse hypothesis is true, is cumulative deterioration that stops just short of death.' (My emphasis)

Yet, in the argument for quantum immortality it is not life per se, but consciousness or self-awareness that plays a role; a branch containing an eternal life in a vegetative state or in coma is no different from one containing death.

The distinction I have just pointed out will be admitted to make a huge difference to the argument for expecting torment once some statistical facts about death are presented. Death as such is usually preceded by a process, however short, of dying. Fred Feldman even proposes a concept of dying2 to account for death as 
a process ${ }^{5}$. Statistically, most cases of death are preceded by a process of dying. The process involves brain death at the future end, but brain death is always preceded by states of unconsciousness. This is true even of deaths that are considered sudden, like death from cardio-respiratory arrest. There is a brief temporal interval in which the victim is not conscious. Other times death is preceded by a longer period of coma or by coma followed by a vegetative state. These can last from a few seconds to several years. All these proximate states of dying are unconscious, hence, following the logic of the argument for quantum immortality, they should be discarded when computing our expectations. Cases of instantaneous deaths are very rare. Some such cases are when, say, a two-ton piece of concrete falls perpendicular on one's head from a certain distance, or, to use Feldman's example, when a butterfly happens to be fluttering in the immediate vicinity of a nuclear blast. Therefore, it is not only death per se, but the vicinity of death as well that is imbued with oblivion.

There are few more statistical facts about dying that we need to make explicit. ${ }^{6}$ Lewis talks about 'cumulative deterioration that stops just short of death' as what we should expect. Now, stopping short of death means suffering a life-threatening condition. Such conditions are most strongly statistically positively correlated with death, and second most strongly with very deep coma. Very deep coma (as measured by the Glasgow Coma Scale) strongly positively correlates with death, again. So from a subjective point of view what we should expect in terms of experiences is not stopping short of death in the sense of making it to the vicinity of death, but rather not making it to that vicinity at all (only to the vicinity of that vicinity), given that the vicinity of death is imbued with oblivion. We should expect not make it as far towards death as to even lose consciousness. In other words, we should not expect to be in a life-threatening condition to begin with.

To be sure, it is compatible with this finding that we should expect to get old and sick, but never close to a resuscitation machine or to intensive care unit, as Lewis' scenario suggest. We would at most be in mild and short coma, a non-life-threatening

5 Fred Feldman, Confrontations with the Reaper: A Philosophical Study of the Nature and Value of Death (Oxford University Press, 1992), chapter 5.

6 They can be checked in a recent study by David Bates, which synthesizes the relevant findings: 'The Prognosis of Medical Coma', Journal of Neurology, Neurosurgery, and Psychiatry 71 (2001), pp. i20-i23. 
condition which is a positive predictor of good recovery, without severe disabilities. To live like this for eternity is definitely not a torment as the one Lewis envisages, but the 'usual decrepitude' if we are to otherwise (i.e. non-quantum-mechanics motivated) live forever. The Terrifying Corollary is thus replaced by the Comforting Corollary.

\section{Interlude: How comforting is this?}

A fair question at this point is whether this scenario is to be called 'comforting' in any sense. It looks as though what I have called the 'usual decrepitude' would no less be considered uncomfortable by any ordinary standards than the quantum mechanics based scenario. Fair enough.

There are two questions to be addressed here. One is whether Lewis's quantum-based scenario of decrepitude is intended, or at least can be understood, as especially nasty, namely, nastier than the scenario of merely aging, as we actually do, but with the difference that it continues indefinitely and without the occurrence of any life-threatening condition. The other issue is whether my 'comforting scenario' is in itself to be preferred to death, or, in other words, whether we should be happy with the truth of the many-worlds account.

To start with the second issue, of course, I don't think we should be happy with the truth of the many-worlds interpretation. Death is preferable, but not because there something especially nasty about the quantum mechanical setting generating immortality. It is because of more general considerations about unhappiness resulting from immortality regardless of what is the reason for being immortal, which is well depicted in various literary works.

Regarding the first issue, there are in Lewis's article both passages that indicate that quantum immortality is something akin to hell, that is, much nastier than mere indefinite aging, and that it is meant simply as aging indefinitely. The former interpretation is to be found in several places, including the second passage I quoted above, where Lewis thinks of quantum immortality as life constantly spent in the vicinity of death, where just barely enough of you to sustain life continues to exist, which situation is relevant not only to life-threatening accidents, but also to 'all the other deadly dangers that we face'. 
Evidence for the latter interpretation is to be found in my first quote, as well as in the passage where Lewis likens the quantum immortality scenario to the situation of the Struldbrugs depicted in Jonathan Swift's Gulliver's Travels, who are immortal but indefinitely undergoing the normal processes of aging. Of course, the situation of the Struldbrugs is far from pleasant, and I'm not sure whether Lewis meant to distinguish it from an even nastier quantum torment of the kind that is prompted by thinking about 'barely enough of you to sustain life', but even if he didn't, the distinction does make sense and seems justified. If so, then my argument is directed against the concern for the likelihood of this 'real torment', the remaining potential disagreement being terminological.

\section{Lucky avoidances}

Now, of course, what the scenario I have argued for in section 2 means is that we will go through an extremely unlikely infinite series of lucky avoidances of the dangers of life, and that is itself perhaps very implausible. The scenario appears as highly unlikely per se, but a moment of inspection shows that it is not really so in the context of comparing it with Lewis's scenario. To illustrate the point, let us use Lewis's own example of how to test the no-collapse hypothesis, namely, imagine that you are crossing the highway at closing time ('How many lives', p. 19), and you have your eyes closed. Lewis's line of thought is that you should expect to unluckily survive infinitely many such crossing attempts, all these attempts being ended by a high frontal impact with a car, and to always wake up in a hospital, in a worse and worse condition. According to my line of thought, you should rather expect to always luckily avoid life-threatening events in infinitely many such crossing attempts, by not being hit (too hard) by a car to begin with. That is so because according to my argument the branching of the world, relevant from the subjective perspective, takes place earlier than it does according to Lewis. According to him it takes place just before the moment of death, according to my reasoning it takes place just before the moment of losing consciousness. Hence, according to my argument, when crossing the highway you should not expect to even lose consciousness, hence, you should not expect to be hit (too hard) by a car at all. So instead of surviving a huge impact, you should expect that the driver swerves 
at the very last moment before impact! In other words, since the subjectively relevant superposition is loss-of-consciousness/no-loss-ofconsciousness, there must be a corresponding superposition at the level of the impact events (dangers of life), and that will be the high-frontal-impact/no-high-frontal-impact superposition.

Which one is likelier: Lewis's scenario or mine? My intuition is that the latter. The reason is that being hit by a car in that situation is extremely likely, so not being hit is extremely unlikely; but, at the same time, once you are hit with high impact the chances of survival are at least as low (if not clearly lower!) as the chances of not having been hit at all, namely, the chances that all the relevant drivers swerved at the right moment when you were crossing the road. And, as a matter of empirical fact, we observe a higher number of successfully swerving drivers than of miraculously surviving pedestrians.

So there is nothing very implausible, or at least more implausible, about my survival scenario, based on perpetual avoidance of dangers, as compared to Lewis' survival scenario, based on perpetual harm resulting from encounters with those dangers.

\section{The problem of miraculous recovery}

There is, however, a possibility in the space of branches compatible with self-awareness that we have missed in the above discussion, namely, the case in which someone suffers a truly life-threatening condition, characterized by deep coma, and nevertheless regains self-awareness and ends up in a condition of severe disability. This is a case of suffering like the ones Lewis envisages. It is a case of making it to the vicinity of death, but escaping, unluckily. And it does actually happen although very rarely. However rarely it happens, according to the argument for quantum immortality it should be taken as a likely outcome if it gets the lion's share of total intensity associated with all the branching scenarios that are compatible with self-awareness. The question is, therefore, whether it really gets the lion's share of the total intensity of conscious branches.

Following Lewis's line of thought according to which after repeated injuries you should find barely enough of yourself to sustain life, and changing 'life' with 'consciousness', we get the result that you should expect immortality with barely enough of you to sustain consciousness. But it is a fact that barely enough of 
you (i.e. neurophysiological basis) to sustain consciousness correlates with barely enough of consciousness. The more damage your brain suffers, the less you are able to suffer. The first level of consciousness after coma and the vegetative state is the so-called Minimally Conscious State, a condition formally recognized in 1995, at the American Congress of Rehabilitation Medicine. ${ }^{7}$ This state can, therefore, be taken as the bare minimum of consciousness for humans. There is some evidence that there is minimal emotional processing in this state, reflected in cortical activity as revealed by MRI and PET studies, ${ }^{8}$ so it looks as though this state could serve as the filler of the role of eternal quantum torment.

However, as we are focusing on being perpetually in the vicinity of death and then escaping, what we should expect is an infinite series of relapses into coma or the vegetative state followed by reemergence into the minimally conscious state. As this infinite process implies perpetual cumulative degradation of the neurophysiological basis of consciousness, we should expect longer and longer periods of unconsciousness coupled with shorter and shorter episodes of minimal consciousness.

Further, it is well documented in the medical literature that emergence from coma or the vegetative state is followed by amnesia, and in the minimal conscious state nothing more in terms of memory than recalling one's name has been shown to be present. ${ }^{9}$ This means that in the long run the shorter and shorter episodes of self-awareness are also mnemonically disconnected from one another. When waking up, you shouldn't remember any of your previous episodes of minimal consciousness. Hence, what we should expect in the long run, from the subject's point of view, if the no-collapse hypothesis is true and our previous hypothesis of never making it to the vicinity of death is false, is not an eternal life of suffering, but rather one extremely brief moment of possibly

7 J.T. Giacino and R. Malone, 'The Vegetative and Minimally Conscious States'. In G.B. Young and E.F.M. Wijdicks (eds.), Handbook of Clinical Neurology. Disorders of Consciousness (Elsevier BV, 2008).

8 T. Bekinsschtein et al., 'Emotion Processing in the Minimally Conscious State', Journal of Neurology, Neurosurgery, and Psychiatry 75 (2004), p. 788, S. Lauries et al., 'Cerebral Processing in the Minimally Conscious State', Neurology 63 (2004), pp. 916-918.

9 R. Nakase-Richardson et al., 'Emergence from Minimally Conscious State', Neurology 73 (2009), pp. 1120-1126. 
painful self-awareness ${ }^{10}$ - call it the 'Momentary Life' scenario. This is also far from Lewis's terrifying corollary.

\section{The problems of miraculous escape and conscious vegetative state}

There are two more possibilities that we should consider. One is that of miraculous escape. Miraculous escape is not the same as miraculous recovery. In my argument I assumed that the loss of consciousness occurs simultaneously with the impact event (the life-threatening quantum accident). If we don't assume that, but rather that loss of consciousness occurs (very shortly) after the impact happens, then we get what I call 'miraculous escape'. In this scenario, first the impact event occurs, and then the consciousness-and-unconsciousness branching occurs immediately after. This means that what I should expect is to suffer the accident, but not even lose my consciousness. In other words, even though the accident is really life threatening (unlike in the scenario entailed by my argument against Lewis), at its completion I emerge without a scratch. Now, such a scenario is a possibility in quantum mechanics, as Lewis himself points out by appeal to the phenomenon of quantum tunneling, but, again, as Lewis himself admits, it looks as the most unlikely of all possible scenarios ('How many lives', p. 18). Hence, it is not a threat to the scenario entailed by my argument.

The second possibility is that of consciousness in the vegetative state. Recent empirical work by Adrian Owen of Cambridge University shows, indeed, that there is likely residual cognitive processing even in the persistent vegetative state, for which it used to be orthodoxy to be defined as not involving any level of consciousness - it used to be understood as 'a state of wakefulness without awareness'. ${ }^{11}$

It looks as though if there is consciousness in the persistent vegetative state, a state that is most likely to occur after coma, but

10 Objectively speaking, of course, the expectation is eternal life, spent almost entirely in an unconscious state.

11 Adrian M. Owen et al., 'Detecting Residual Cognitive Function in Persistent Vegetative State (PVS)', Neurocase 8 (2002), pp. 394-403, A. M. Owen, et al., 'Residual Auditory Function in Persistent Vegetative State: A combined PET and fMRI study', Neuropsychological Rehabilitation 15(2005), pp. 290-306. Thanks to Katalin Farkas for raising the objection and calling my attention to Owen's work. 
can also occur right after a life-threatening accident, then this state can also serve as a version of Lewis's torment since it might involve the capacity of the victim to suffer. The persistent vegetative state is likely enough to occur after life-threatening events, hence, we can't appeal to intrinsic improbability as in the case of miraculous escape.

However, in order for the conscious vegetative state to threaten my argument, one needs to have empirical evidence that there is consciousness from the very beginning of the occurrence of that state, that is, immediately after the accident occurs, so that loss of consciousness does not occur at all. Only that way one could argue to the likelihood ending up in that state, from the subjective point of view, as the accident occurs. Otherwise, if consciousness occurs later and after an episode of unconsciousness, then we are back to the argument for the scenario entailed by miraculous recovery (the Momentary Life scenario).

\section{Conclusion}

We are left with two likely scenarios of what we should expect as regards our future immortal life if the many-worlds interpretation of quantum mechanics is the right one: the Comforting Corollary and Momentary Life. We can't assess whether one or the other gets the lion's share of the total intensity associated with branches compatible with self-awareness, but we can be sure that they together (i.e. their disjunction) do indeed get the lion's share, which is much reassuring.

Bilkent University, 06800 Bilkent, Ankara, Turkey aranyosi@bilkent.edu.tr istvanaranyosi@gmail.com 